Proc. Estonian Acad. Sci. Biol. Ecol., 2005, 54, 4, 253-254

\title{
New enchytraeid species since 2002
}

\author{
Rüdiger M. Schmelz ${ }^{a^{*}}$ and Jörg Römbke ${ }^{b}$ \\ a Department of Animal Biology, Plant Biology and Ecology, Faculty of Sciences, University of \\ La Coruña, Alejandro da Sota 1, 15008 La Coruña, Spain \\ b ECT Oekotoxicologie GmbH, Böttgerstr. 2-14, D-65439 Flörsheim, Germany \\ Received 17 December 2004
}

Abstract. A list of 21 enchytraeid species (Oligochaeta, Enchytraeidae) new to science described in 2002-2005 is presented.

Key words: enchytraeids, new species.

This list of new species continues the lists of new enchytraeid taxa published in previous issues of the Newsletter on Enchytraeidae.

Achaeta unibulba Graefe, Dózsa-Farkas \& Christensen, 2005

Cernosvitoviella tridentina Dumnicka, 2004

Fridericia argillae Schmelz, 2003

Fridericia auritoides Schmelz, 2003

Fridericia composti Schmelz, 2003

Fridericia cusanica Schmelz, 2003

Fridericia dozsae Schmelz, 2003

Fridericia eiseni Dózsa-Farkas, 2005

Fridericia granosa Schmelz, 2003

Fridericia healyae Schmelz, 2003

Fridericia larix Schmelz \& Collado, 2005

Fridericia lenta Schmelz, 2003

Grania aquitana Rota \& Erséus, 2003

Grania canaria Rota \& Erséus, 2003

Grania fortunata Rota \& Erséus, 2003

Grania mauretanica Rota \& Erséus, 2003

Grania papillinasus Rota \& Erséus, 2003

* Corresponding author, schmelz@vodafone.es 
Grania torosa Rota \& Erséus, 2003

Grania vikinga Rota \& Erséus, 2003

Marionina sexdiverticulata Dózsa-Farkas, 2002

Marionina spongicola Rota \& Manconi, 2004

\title{
REFERENCES
}

Dózsa-Farkas, K. 2002. The enchytraeid fauna (Annelida, Oligochaeta: Enchytraeidae) of the FertöHanság National Park. In The Fauna of the Fertö-Hanság National Park, pp. 153-163. Hungarian Natural History Museum, Budapest.

Dózsa-Farkas, K. 2005. Fridericia eiseni sp. n., a new enchytraeid species from the Fridericia ratzeli species group. Proc. Estonian Acad. Sci. Biol. Ecol., 54, 279-291.

Dumnicka, E. 2004. A description of Cernosvitoviella tridentina, a new species of Enchytraeidae (Oligochaeta) from the Italian Alps. Ann. Limnol., 40, 133-137.

Graefe, U., Dózsa-Farkas, K. \& Christensen, B. 2005. Achaeta unibulba sp. n., a widespread European species (Oligochaeta, Enchytraeidae). Proc. Estonian Acad. Sci. Biol. Ecol., 54, 271-278.

Rota, E. \& Erséus, C. 2003. New records of Grania (Clitellata, Enchytraeidae) in the Northeast Atlantic (from Tromsø to the Canary Islands), with descriptions of seven new species. Sarsia, 88, 210-243.

Rota, E. \& Manconi, R. 2004. Taxonomy and ecology of sponge-associate Marionina spp. (Clitellata: Enchytraeidae) from the Horomatangi Geothermal System of Lake Taupo, New Zealand. Int. Rev. Hydrobiol., 89, 58-67.

Schmelz, R. M. 2003. Taxonomy of Fridericia (Oligochaeta, Enchytraeidae). Revision of species with morphological and biochemical methods. Abh. naturwiss. Ver. Hamburg (NF), 38.

Schmelz, R. M. \& Collado, R. 2005. Fridericia larix (Enchytraeidae, Oligochaeta) from Irish soils. Org. Div. Evolut., 5, 85-88.

\section{Uued valgeliimuklaste liigid pärast aastat 2002}

\author{
Rüdiger M. Schmelz ja Jörg Römbke
}

Nimestik aastail 2002-2005 kirjeldatud 21 teadusele uuest valgeliimuklaste (Oligochaeta, Enchytraeidae) liigist. 